\title{
Filippov-Pliss lemma for dynamical inclusions on a time scale
}

\author{
M Rafaqat ${ }^{*}$ (D, R Ahmed ${ }^{2}$, T Donchev ${ }^{3}$ and V Lupulescu ${ }^{4}$
}

"Correspondence:

m.rafaqat50@gmail.com

'Department of Mathematics, University of Lahore, Lahore,

Pakistan

Full list of author information is available at the end of the article

\begin{abstract}
In this paper we prove two variants of the well-known Filippov-Pliss lemma in the case of dynamical inclusions on a time scale. The first variant is when the right-hand side is Lipschitz continuous on the state variable. Afterward we introduce one-sided Perron conditions for multifunctions on a time scale and prove the second variant of that lemma. Some discussions on relaxed systems are presented.
\end{abstract}

MSC: Primary 34N05; secondary 39N99

Keywords: dynamical inclusions on a time scale; one-sided Perron condition; Filippov-Pliss lemma

\section{Introduction}

Time scale theory is introduced in $[1,2]$ in order to unify the continuous and discrete systems. We refer the reader to $[3,4]$ for the theory of dynamical equations on a time scale and [5, 6], where some applications are given. Among others notice [7] where the theory of dynamical systems in measure chains is studied. In [8] the integration on a time scale is investigated and its connections with standard Lebesgue integral are considered in [9].

In the last years different optimization problems on time scales have been studied. We refer the reader to [10-13]. The theory of dynamical inclusions on a time scale is presented in [14-16]. In the later paper [17] the authors prove the analogy of Filippov's selection theorem, which shows that the optimal control of Caratheodory controlled systems can be written equivalently as differential inclusions.

One of the most useful results in optimal control is the significant lemma of FilippovPliss (see, e.g., [18]). It was proved first time by Filippov in the case of Lipschitzian righthand side in [19] and afterward extended by Pliss in [20] under much weaker conditions. We refer the reader to [21, 22] for the main applications of this result. Notice also [23] where the history and review of this lemma is presented. This result has been extended to the case of one-sided Lipschitz differential inclusions in [24].

We refer the reader to $[25,26]$ for the needed facts in set-valued analysis and differential inclusions.

In this paper we prove a variant of this lemma in the case of Lipschitz dynamical inclusion. Afterward one-sided Perron condition is introduced in the case of a time scale, and a variant of Filippov-Pliss lemma is proved in the case of one-sided Perron inclusions on a

(c) The Author(s) 2017. This article is distributed under the terms of the Creative Commons Attribution 4.0 International License (http://creativecommons.org/licenses/by/4.0/), which permits unrestricted use, distribution, and reproduction in any medium, provided you give appropriate credit to the original author(s) and the source, provide a link to the Creative Commons license, and indicate if changes were made. 
time scale. One of the most important theorems in the optimal control is that under some additional hypotheses the closure of the solution set of the original system is the solution set of the convexified one. We discuss its extension on dynamical inclusions.

The paper is organized as follows. In the next section the needed definitions, notations and preliminary results are given. Third section deals with system description. The main results of the presented paper are given in the fourth section, where we study the FilippovPliss lemma and relaxation theorem. Finally, we present conclusion with discussion of other possibilities for the definition of one-sided Perron condition.

\section{Preliminaries}

Recall that (see, e.g., [3]) every closed subset $\mathbb{T} \subset \mathbb{R}$ is called a time scale, and hence the time scale $\mathbb{T}$ is a complete metric space with the usual metric on $\mathbb{R}$. Furthermore, the intersection of $\mathbb{T}$ with any closed bounded interval is a compact set.

Now we recall the main fact that we will use. Let $t \in \mathbb{T}$, the forward jump operator $\sigma$ : $\mathbb{T} \rightarrow \mathbb{T}$ is defined as follows:

$$
\sigma(t)=\inf \{s \in \mathbb{T}: s>t\}
$$

Let $\mu(t)=\sigma(t)-t$, clearly $\mu(t)=0$ if $t$ is right dense point. The point $t \in \mathbb{T}$ is said to be right scattered if $\mu(t)>0$. Denote by $\mathbb{T}_{\text {rd }}$ the right dense and by $\mathbb{T}_{\text {rs }}$ the right scattered point of $\mathbb{T}$, then $\mathbb{T}_{\mathrm{rd}} \cap \mathbb{T}_{\mathrm{rs}}=\emptyset$. Analogously, $\rho(t)=\sup \{s \in \mathbb{T}: s<t\}$. The point $t$ is said to be left scattered if $\rho(t)<t$. The set $\mathbb{T}^{\kappa}$ is defined as follows: If $\mathbb{T}$ has a left scattered maximum $S$, then $\mathbb{T}^{\kappa}=\mathbb{T} \backslash\{S\}$; otherwise, it coincides with $\mathbb{T}$.

Let $f: \mathbb{T} \longrightarrow \mathbb{R}^{n}$ and let $t \in \mathbb{T}$. Suppose there exists $A \in \mathbb{R}^{n}$ with the property: for every $\epsilon>0$, there exists $\delta>0$ such that

$$
|f(\sigma(t))-f(s)-A(\sigma(t)-s)| \leq \epsilon|\sigma(t)-s|
$$

for every $s \in(t-\delta, t+\delta) \cap \mathbb{T}$. The vector $A$ is called $\triangle$-derivative and is denoted by $f^{\Delta}(t)$.

Now we recall some properties of the $\Delta$-derivative.

Proposition 2.1 Let $f: \mathbb{T} \rightarrow \mathbb{R}^{n}$ and $t \in \mathbb{T}^{k}$.

(a) Iff is $\Delta$-differentiable at $t$, then $f$ is continuous at $t$.

(b) Iff is continuous at $t \in \mathbb{T}_{\mathrm{rs}}$, then

$$
f^{\Delta}(t)=\frac{f(\sigma(t))-f(t)}{\mu(t)} .
$$

(c) The mapf is $\Delta$-differentiable at $t \in \mathbb{T}^{k} \backslash \mathbb{T}_{\mathrm{rs}}$ if and only if

$$
f^{\Delta}(t)=\lim _{s \rightarrow t} \frac{f(t)-f(s)}{t-s} .
$$

(d) Let $f, g: \mathbb{T} \rightarrow \mathbb{R}^{n}$ be $\Delta$-differentiable, then the scalar product $\langle f, g\rangle$ is also $\Delta$-differentiable and

$$
\langle f(t), g(t)\rangle^{\Delta}=\left\langle f(t), g^{\Delta}(t)\right\rangle+\left\langle f^{\Delta}(t), g(t+\mu(t))\right\rangle .
$$


In particular,

$$
\left\langle f^{2}(t)\right\rangle^{\Delta}=\left\langle f(t)+f(t+\mu(t)), f^{\Delta}(t)\right\rangle .
$$

It follows from Proposition 2.1 that $f(\sigma(t))=f(t)+\mu(t) f^{\Delta}(t)$. Evidently a vector-valued function $f(\cdot)$ is $\Delta$-differentiable at $t$ iff every coordinate function $f_{i}(\cdot)$ is $\Delta$-differentiable at $t$ and $f^{\Delta}(t)=\left(f_{1}^{\Delta}(t), \ldots, f_{n}^{\Delta}(t)\right)$.

Denote the interval $\mathbb{I}=\left[t_{0}, T\right)_{\mathbb{T}}=\{\tau \in \mathbb{T}: a \leq \tau<b\}$. The outer measure of the set $A \subset \mathbb{T}$ is

$$
m^{*}(A)=\inf \left[\sum_{i=1}^{\infty}\left(b_{i}-a_{i}\right): A \subset \bigcup_{i}\left[a_{i}, b_{i}\right)\right] .
$$

The set $A \subset \mathbb{T}$ is said to be $\Delta$-measurable if

$$
m^{*}(A)=m^{*}(A \cap B)+m^{*}(A \cap(\mathbb{T} \backslash B))
$$

for every subset $B \subset \mathbb{T}$.

Definition 2.2 The function $f: \mathbb{T} \rightarrow \mathbb{R}^{n}$ is said to be $\Delta$-measurable if, for every open set $B \subset \mathbb{R}^{n}$, the set

$$
f^{-1}(B)=\{t \in \mathbb{T}: f(t) \in B\}
$$

is $\Delta$-measurable.

The multifunction $F: \mathbb{T} \rightrightarrows \mathbb{R}^{n}$ is said to be $\Delta$-measurable if, for every compact set $B \subset$ $\mathbb{R}^{n}$,

$$
F^{-1}(B)=\{t \in \mathbb{T}: F(t) \cap B \neq \emptyset\}
$$

is $\Delta$-measurable.

We notice the following property:

If $A_{1} \cap A_{2}=\emptyset$ are $\Delta$-measurable and $A=A_{1} \cup A_{2}$, then the multifunction $H: A \rightrightarrows \mathbb{R}^{n}$

is $\Delta$-measurable if and only if $H$ is $\Delta$-measurable as a map from $A_{i}$ into $\mathbb{R}^{n}$ for $i=1,2$.

Furthermore, every $\Delta$-measurable function $f(\cdot)$ satisfies Lusin's property, i.e., there exists a sequence of pairwise disjoint closed sets $\mathbb{I}_{n} \subset \mathbb{I}$ such that the $\Delta$-measure of $\mathbb{I} \backslash \bigcup_{m=1}^{\infty} \mathbb{I}_{m}$ is 0 and $f$ is continuous on $\mathbb{I}_{k} \times \mathbb{R}^{n}$ for every $k \geq 1$.

Definition 2.3 A real-valued function $f:[a, b] \longrightarrow \mathbb{R}^{n}$ defined on $[a, b]$ is said to be absolutely continuous if, for every $\epsilon>0$, there exists $\delta>0$ such that $\sum_{k=1}^{n}\left|f\left(b_{k}\right)-f\left(a_{k}\right)\right|<\epsilon$ for every $n$ disjoint subintervals $\left(a_{k}, b_{k}\right)$ of $[a, b], a_{k}<b_{k}, k=1,2, \ldots, n$, such that $\sum_{k=1}^{n} \mid b_{k}-$ $a_{k} \mid<\delta$.

Proposition 2.4 (Proposition 2.13 in [15]) If the function $f: \mathbb{I} \rightarrow \mathbb{R}^{n}$ is absolutely continuous, then the $\Delta$-measure of the set $\left\{t \in \mathbb{I}_{\mathrm{rd}}: f(t)=0\right.$ and $\left.f^{\Delta}(t) \neq 0\right\}$ is zero. 
As it is well known (see, e.g., [26]) every $\Delta$-measurable multifunction $F(\cdot)$ admits $\Delta$ measurable selection $f(t) \in F(t)$.

Definition 2.5 The multifunction $F: \mathbb{I} \times \mathbb{R}^{n} \rightrightarrows \mathbb{R}^{n}$ is said to be

- Upper semi-continuous (USC) at $(\tau, y)$ if, for every $\varepsilon>0$, there exists $\delta$ such that $F((\tau-\delta, \tau+\delta) \cap \mathbb{I}, x+\delta \mathbb{B}) \subset F(\tau, y)+\varepsilon \mathbb{B}$, where $\mathbb{B}$ is the closed unit ball.

- Lower semi-continuous (LSC) at $(\tau, y)$ if, for every $\mathbb{I} \ni t_{i} \rightarrow \tau, x_{i} \rightarrow y$ and $f \in F(\tau, y)$, there exists $f_{i} \in F\left(t_{i}, x_{i}\right)$ with $f_{i} \rightarrow f$.

- Continuous if it is simultaneously USL and LSC.

\section{System description}

We study the following dynamical inclusion on a time scale:

$$
x^{\Delta} \in F(t, x(t)), \quad \Delta \text {-a.e. } t \in \mathbb{I}, x\left(t_{0}\right)=x_{0},
$$

where $F: \mathbb{I} \times \mathbb{R}^{n} \rightrightarrows \mathbb{R}^{n}$ is with nonempty convex compact values. Notice that $\mathbb{I}=\mathbb{I}_{\mathrm{rd}} \cup \mathbb{I}_{\mathrm{rs}}$.

The $\Delta$-absolute continuous function $x(\cdot)$ is said to be a solution of (3.1) if it satisfies inclusion (3.1) for $\Delta$ a.a. $t \in \mathbb{I}$.

Denote by $\mathcal{B}$ the Borel $\sigma$ algebra on $\mathbb{R}^{n}$.

Definition 3.1 The multivalued map $F: \mathbb{I} \times \mathbb{R}^{n} \rightrightarrows \mathbb{R}^{n}$ is said to be $\Delta \times \mathcal{B}$ measurable if, for every open set (and hence $\mathcal{B}$ measurable) $B \subset \mathbb{R}^{n}$, the set

$$
F^{-1}(B)=\left\{(t, u) \in \mathbb{I} \times \mathbb{R}^{n}: F(t, u) \cap B \neq \emptyset\right\} \text { is } \Delta \times \mathcal{B} \text { measurable. }
$$

F1. $|F(t, x)| \leq \lambda(1+|x|)$ (sublinear growth), where $\lambda$ is a positive constant.

Remark 3.2 It is standard to show that under F1 there exist constants $M$ and $N$ such that $|x(t)| \leq M$ and $\|F(t, x(t)+\mathbb{B})+\mathbb{B}\| \leq N$ for every $t \in \mathbb{T}$ and for every solution $x(\cdot)$ of

$$
x^{\Delta}(t) \in F(t, x(t)+\mathbb{B})+\mathbb{B}, \quad x\left(t_{0}\right)=x_{0}
$$

when such a solution exists.

F2. The map $F(\cdot, \cdot)$ is $\Delta \times \mathcal{B}$ measurable and, for every $t \in \mathbb{I}$, the map $F(t, \cdot)$ is USC.

We are going to prove the existence theorem, because we did not see the following variant of it. For other existence results, we refer the reader to $[15,16]$.

Theorem 3.3 Under F1, F2 system (3.1) has a solution. The solution set is $C\left(I_{\mathbb{I}}, \mathbb{R}^{n}\right)$ compact.

Proof Fix $h_{n}>0$. For $t>t_{0}$, we define the approximate solution $x_{n}(\cdot)$ as follows:

$$
x_{n}(t)=x\left(t_{0}\right)+\int_{t_{0}}^{t} f_{n}(s) d s,
$$

where $f_{n}(t) \in F\left(t, x_{n}\left(t_{0}\right)\right)$. If $t_{0}+h_{n} \in \mathbb{I}$, then we replace $t_{0}$ by $t_{0}+h_{n}$ when it is less than $T$ and continue as in (3.2) for $t>t_{0}+h_{n}$. If $t_{0}+h_{n} \notin \mathbb{I}$, then there exists $\tau \in \mathbb{I}$ such that $t_{0}+h_{n} \in$ 
$(\tau, \sigma(\tau))$. Then we take $x_{n}(\sigma(\tau))=x_{n}(\tau)+x_{n}^{\Delta}(\tau) \cdot \mu(\tau)$, where $x_{n}^{\Delta} \in F\left(\tau, x_{n}(\tau)\right)$. Then we replace $t_{0}$ by $\sigma(\tau)$ and continue as in (3.2) for $t>\sigma(\tau)$. Clearly, one can define $x_{n}(\cdot)$ on the whole interval $\mathbb{I}$ thanks to the growth condition F1. The sequence $\left\{x_{n}(\cdot)\right\}_{n=1}^{\infty}$ is uniformly bounded and equicontinuous. In fact we have $x_{n}^{\Delta}(t) \in F\left(t, x_{n}(t)+\mathbb{B}\right)$ and $\left\|x_{n}^{\Delta}(t)\right\| \leq N$ (see Remark 3.2 mentioned above). We claim that $x_{n}^{\Delta} \in F\left(t, x_{n}(t)+\varphi_{n}(t) \mathbb{B}\right)$, where $\varphi_{n} \rightarrow 0 \Delta$ a.e. on $\left[t_{0}, T\right)_{\mathbb{T}}$. Due to our construction $t \in\left[\tau, \tau^{\prime}\right)_{\mathbb{T}}$,

$$
x_{n}(t)=x_{n}(\tau)+\int_{\tau}^{t} f_{n}(s) d s
$$

where $f_{n}(s) \in F\left(s, x_{n}(\tau)\right)$. We have two cases as follows.

Case (i): If $\tau \in \mathbb{T}_{\text {rd }}$, then $\tau^{\prime}-\tau \leq h_{n}$, i.e., $|x(t)-x(\tau)| \leq N h_{n}$, where $N$ is the constant from Remark 3.2. Therefore $x_{n}^{\Delta}(t) \in F\left(t, x_{n}(t)+N h_{n}\right)$.

Case (ii): If $\tau \in \mathbb{T}_{\mathrm{rs}}$, then $\tau^{\prime}=\sigma(\tau)$. In this case $\left[\tau, \tau^{\prime}\right) \cap \mathbb{T}=\tau$ and hence $t=\tau$. However, $x_{n}^{\Delta}(t) \in F\left(t, x_{n}^{\Delta}(t)\right)$. The claim is proved because $\lim _{h_{n} \rightarrow 0} N h_{n}=0$.

From Theorem 3.5 of [17] we know that there exists a subsequence $x_{n_{k}}(\cdot)$ which converges uniformly to a solution $x(\cdot)$ of (3.1). The proof is therefore complete.

Recall that the multimap $F(\cdot, \cdot)$ is said to be almost USC (LSC, continuous) if, for every $\varepsilon>0$, there exists a set $N_{\varepsilon} \subset \mathbb{I}$ with $\Delta$ measure less than $\varepsilon$ and such that $F(\cdot, \cdot)$ is USC (LSC, continuous) on $\left(\mathbb{I} \backslash I_{\varepsilon}\right) \times \mathbb{R}^{n}$.

We can prove the following proposition.

Proposition 3.4 Let $F(\cdot, \cdot)$ have nonempty convex compact values, and let it be almost USC, then $F$ is $\Delta \times \mathcal{B}$ measurable and $F(t, \cdot)$ is USC for $\Delta$ a.a. $t \in \mathbb{I}$.

Proof It is easy to see that $F(\cdot, \cdot)$ is almost USC iff there exists a sequence of pairwise disjoint closed sets $\mathbb{I}_{n} \subset \mathbb{I}$ such that $\Delta$-measure of $\mathbb{I} \backslash \bigcup_{m=1}^{\infty} \mathbb{I}_{m}$ is 0 and $F$ is USC on $\mathbb{I}_{k} \times \mathbb{R}^{n}$ for every $k \geq 1$. Therefore $F$ is $\Delta \times \mathfrak{B}$ measurable on $\mathbb{I}_{k} \times \mathbb{R}^{n}$ for every $k \geq 1$ and hence on $\mathbb{I} \times \mathbb{R}^{n}$. Also $F(t, \cdot)$ is USC for $\Delta$ a.e. $t$.

\section{Filippov-Pliss lemma on a time scale}

In this section we prove the main results in the paper. We prove two versions of the Filippov-Pliss lemma which have many applications in optimal control (cf. [18]).

We need the following result, which is a particular case of Proposition 1.43 of [25].

Proposition 4.1 Let $F, G: \mathbb{I} \times \mathbb{R}^{n} \rightrightarrows \mathbb{R}^{n}$ be $\Delta \times \mathcal{B}$ measurable and at least one with compact values. Then the map $H(t, x)=F(t, x) \cap G(t, x)$ is also $\Delta \times \mathcal{B}$ measurable.

Now we will prove two variants of the Filippov-Pliss theorem for dynamical inclusion on a time scale. The first proof deals with Lipschitz right-hand side.

Theorem 4.2 Let $F(\cdot, \cdot)$ satisfy $\mathbf{F 1}, \mathbf{F 2}$, and let $F(t, \cdot)$ be L-Lipschitz. Iff $(\cdot)$ is a $\Delta$-integrable function on $\mathbb{I}$ and if $y(\cdot)$ is an ACfunction with

$$
\operatorname{dist}\left(y^{\Delta}(t), F(t, y(t))\right) \leq f(t)
$$

then there exists a solution $x(\cdot)$ of (3.1) such that $|x(t)-y(t)| \leq r(t)$, where $r^{\Delta}(t)=\operatorname{Lr}(t)+f(t)$ and $r\left(t_{0}\right)=\left|x_{0}-y_{0}\right|$. Furthermore, $\left|x^{\Delta}(t)-y^{\Delta}(t)\right| \leq \operatorname{Lr}(t)+f(t)$. 
Proof Define the map

$$
G(t, u)=\left\{v \in F(t, u):\left|y^{\Delta}(t)-v\right| \leq L|y(t)-u|+f(t)\right\} .
$$

We claim that $G(\cdot, \cdot)$ satisfies $\mathbf{F} \mathbf{1}$ and $\mathbf{F} \mathbf{2}$.

Namely, $G(t, u)$ admits nonempty values because $F(t, \cdot)$ is Lipschitz and it is with nonempty convex compact values. We are going to prove that $G(t, u)$ is closed and convex. Indeed if $v_{i} \in F(t, u)$ and $v_{i} \rightarrow v$, we know that $\left|v-y^{\Delta}(t)\right|=\lim _{i \rightarrow \infty}\left|v_{i}-y^{\Delta}(t)\right|$. Therefore, if $v_{1}, v_{2} \in G(t, u)$, then we have that $\left|y^{\Delta}(t)-\lambda v_{1}-(1-\lambda) v_{2}\right| \leq \lambda\left|y^{\Delta}(t)-v_{1}\right|+(1-\lambda) \mid y^{\Delta}(t)-$ $v_{2}|\leq L| y(t)-u \mid+f(t), \forall \lambda \in(0,1)$. Also $G(t, u)$ is USC. For this, it is enough to see that $G(t, \cdot)$ has a closed graph. Let $v_{i} \in G\left(t, u_{i}\right), u_{i} \rightarrow u$ and $v_{i} \rightarrow v$. Since $F(t, \cdot)$ is USC, one has that $\lim _{i \rightarrow \infty} v_{i}=v \in F(t, u)$. Furthermore, $\left|v_{i}-y^{\Delta}(t)\right| \leq L\left|y(t)-u_{i}\right|+f(t)$, and hence $\left|v-y^{\Delta}(t)\right| \leq L|y(t)-u|+f(t)$.

Now, we have to show that $G(\cdot, \cdot)$ is $\Delta \times \mathcal{B}$ measurable. Let $X \in \mathbb{R}^{n}$. Since $y(\cdot)$ is AC, then $y^{\Delta}(\cdot)$ is $\Delta$-measurable, and hence the multimap $H(t, X)=\left\{(t, z) \in\left[t_{0}, T\right)_{\mathbb{T}} \times \mathbb{R}^{n}: \mid z-\right.$ $\left.y^{\Delta}(t)|\leq L| y(t)-X \mid+f(t)\right\}$ is $\Delta \times \mathcal{B}$ measurable. Due to Proposition 4.1, the map $G(t, X)=$ $H(t, X) \cap F(t, X)$ is also $\Delta \times \mathcal{B}$ measurable. The claim is therefore proved. It follows from Theorem 3.3 that $x^{\Delta} \in G(t, x(t)), x\left(t_{0}\right)=x_{0}$ admits a solution $x(\cdot)$.

From Theorem 1.67 of [3] we know that $|x(t)-y(t)|=r(t)$, where $r^{\Delta}(t) \leq \operatorname{Lr}(t)+f(t)$ for $\Delta$-a.e. $t$ and $r\left(t_{0}\right)=\left|x_{0}-y_{0}\right|$.

The definition of $G(\cdot, \cdot)$ then implies the last statement of the theorem.

Now we will prove a Filippov-Pliss type theorem under much weaker condition, which gives the estimation only of the difference between $x(\cdot)$ and $y(\cdot)$ but not between their derivatives.

Definition 4.3 The multivalued map $F: \mathbb{I} \times \mathbb{R}^{n} \rightrightarrows \mathbb{R}^{n}$ is said to be OSP (one-sided Perron) (on the state variable) if there exists a Perron function $w(\cdot, \cdot)$ such that:

For every $x, y \in \mathbb{R}^{n}$, almost every $t \in \mathbb{I}$ and every $f_{x} \in F(t, x)$, there exists $f_{y} \in F(t, y)$ such that

$$
\begin{aligned}
& \left\langle x-y, f_{x}-f_{y}\right\rangle \leq \frac{1}{2} w(t,|x-y|)|x-y|, \quad \text { if } t \in \mathbb{T}_{\mathrm{rd}} \\
& \left|f_{x}-f_{y}\right| \leq w(t,|x-y|), \quad \text { if } t \in \mathbb{T}_{\mathrm{rs}} .
\end{aligned}
$$

Recall that the function $v(\cdot, \cdot)$ is said to be a Perron function if

- $v(\cdot, \cdot)$ is $\Delta \times \mathcal{B}$ measurable, $v(t, \cdot)$ is continuous;

- $v(\cdot, \cdot)$ is $\Delta$-integrally bounded on the bounded sets and $v(t, 0)=0$;

- the unique solution of $r^{\Delta}(t)=v(t, r(t)), r\left(t_{0}\right)=0$ is $r(t)=0$.

$v(\cdot, \cdot)$ is called module if it satisfies only the first two conditions, but not necessarily the third one.

The definition of OSP condition on a time scale is different than in ordinary differential inclusions. Here it depends also on the point $t$.

Now we extend the previous theorem to the case of OSP multifunctions.

Theorem 4.4 Let $F(\cdot, \cdot)$ satisfy $\mathbf{F 1}, \mathbf{F 2}$, and let $F(t, \cdot)$ be OSP w.r.t. a Perron function $w(\cdot, \cdot)$. If $f(\cdot)$ is a $\Delta$-integrable function on $\mathbb{I}$ and if $y(\cdot)$ is an ACfunction with $\operatorname{dist}\left(y^{\Delta}(t), F(t, y(t))\right) \leq$ 
$f(t)$, then there exists a solution $x(\cdot)$ of (3.1) such that $|x(t)-y(t)| \leq r(t)$, where $r^{\Delta}(t)=$ $w(t, r(t))+f(t)$ and $r\left(t_{0}\right)=\left|x_{0}-y_{0}\right|$.

Proof Clearly, the set-valued map $t \rightarrow y^{\Delta}(t)+f(t) \mathbb{B}$ is $\Delta$-measurable. Therefore $H(t)=$ $F(t, y(t)) \cap\left(y^{\Delta}(t)+f(t) \mathbb{B}\right)$ is also $\Delta$-measurable and hence $t \rightarrow F(t, y(t))$ is $\Delta$-measurable. Thus there exists a $\Delta$-measurable selection $h(t) \in H(t)$. Evidently $h(t) \in F(t, y(t))$.

Now we define the following multifunction:

$$
\begin{aligned}
& G(t, u)=\{v \in F(t, u)\} \quad \text { such that } \\
& \begin{cases}\langle y(t)-u, h(t)-v\rangle \leq w(t,|x-y|)|x-y|, & t \text { is right dense } \\
|h(t)-v| \leq w(t,|x-y|), & t \text { is right scattered. }\end{cases}
\end{aligned}
$$

We claim that $G(t, \cdot)$ is upper semi-continuous for every $t \in \mathbb{I}$. Indeed we have to prove that the graph of $G(t, \cdot)$ is compact. However, the graph is bounded, and hence it remains to show that it is closed.

Let $u_{i} \rightarrow u, v_{i} \in G\left(t, u_{i}\right)$ and $v_{i} \rightarrow v$. We have to show that $v \in G(t, u)$. Clearly $v \in F(t, u)$ because $F(t, \cdot)$ is USC. If $t$ is right dense, then $\left\langle y(t)-u_{i}, h(t)-v_{i}\right\rangle \rightarrow\langle y(t)-u, h(t)-v\rangle, \mid y(t)-$ $u_{i}|\rightarrow| y(t)-u \mid$ and $w\left(\left|y(t)-u_{i}\right|\right) \rightarrow w(t,|y(t)-u|)$. Thus $\langle y(t)-u, h(t)-v\rangle \leq w(t, \mid y(t)-$ $u \mid)|y(t)-u|$, i.e., $v \in G(t, u)$. If $t$ is right scattered, then $|h(t)-v| \leq w(t,|y(t)-u|)$ because $\lim _{i \rightarrow \infty}\left|h(t)-v_{i}\right|=|h(t)-v|$.

We have to prove that $G(\cdot, \cdot)$ is $\Delta \times \mathcal{B}$ measurable.

Consider first the case $G: \mathbb{T}_{\mathrm{rs}} \times \mathbb{R}^{n} \rightrightarrows \mathbb{R}^{n}$. Since $w(t, \cdot)$ and $y(\cdot)$ are continuous as well as $h(\cdot)$ is $\Delta$-measurable, one has that $S(t, u)=\left\{v \in \mathbb{R}^{n}:|h(t)-v| \leq w(t,|y(t)-u|)\right\}$ is $\Delta \times \mathcal{B}$ measurable. Then $\bar{G}(t, u)=F(t, u) \cap \bar{S}(t, u)$ is $\Delta \times \mathcal{B}$ measurable.

Let $G: \mathbb{T}_{\text {rd }} \times \mathbb{R}^{n} \rightrightarrows \mathbb{R}^{n}$. It is easy to see that the map

$$
S(t, u)=\left\{v \in \mathbb{R}^{n}:\langle y(t)-u, h(t)-v\rangle \leq w(t,|y(t)-u|)|y(t)-u|\right\}
$$

is $\Delta \times \mathcal{B}$ measurable.

Therefore $\bar{G}(t, u)=F(t, u) \cap \bar{S}(t, u)$ is also $\Delta$-measurable. Consequently, $G(\cdot, \cdot)$ is $\Delta \times \mathcal{B}$ measurable.

Due to Theorem 3.3, the system

$$
\dot{x} \in G(t, x(t)), \quad x\left(t_{0}\right)=x_{0}
$$

has a solution $x(\cdot)$. Therefore

$$
\left\langle y(t)-x(t), y^{\Delta}(t)-x^{\Delta}(t)\right\rangle \leq|y(t)-x(t)|\left|y^{\Delta}(t)-h(t)\right|+w(t,|y(t)-x(t)|)|y(t)-x(t)|,
$$

$\forall t \in \mathbb{T}_{\text {rd }}$, i.e., $\left(|y(t)-x(t)|^{2}\right)^{\Delta}=2|x(t)-y(t)| \cdot(|x(t)-y(t)|)^{\Delta} \leq 2|y(t)-x(t)|(f(t)+w(t, \mid y(t)-$ $x(t) \mid)$. Clearly $t \rightarrow|x(t)-y(t)|$ is $\Delta$-AC.

From Proposition 2.4 we know that the intersection of the sets $\{t \in \mathbb{T}:|x(t)-y(t)|=0\}$ and $\left\{t \in \mathbb{T}:\left|x^{\Delta}(t)-y^{\Delta}(t)\right| \neq 0\right\}$ has $\Delta$-measure zero. Thus $|x(t)-y(t)|^{\Delta} \leq w(t,|y(t)-x(t)|)+$ $f(t)$ for $\Delta$ almost every $t \in \mathbb{T}_{\text {rd }}$.

If $t \in \mathbb{T}_{\mathrm{rs}}$, then $\left|y^{\Delta}(t)-x^{\Delta}(t)\right| \leq\left|y^{\Delta}(t)-h(t)\right|+\left|h(t)-x^{\Delta}(t)\right| \leq w(t,|y(t)-x(t)|)$. However, $|x(t)-y(t)|^{\Delta} \leq\left|x^{\Delta}(t)-y^{\Delta}(t)\right|$ and hence $|x(t)-y(t)|^{\Delta} \leq w(t,|x(t)-y(t)|)+f(t)$. 


\section{Consequently,}

$$
|x(t)-y(t)| \leq r(t) \text {, where } r^{\Delta}(t)=w(t, r(t))+f(t), r\left(t_{0}\right)=\left|x_{0}-y_{0}\right| .
$$

The proof is therefore complete.

Remark 4.5 It is easy to see that Theorem 4.2 remains true also when $w(\cdot, \cdot)$ is only module.

As it is well known,

$$
\begin{aligned}
\left(\|x(t)\|^{2}\right)^{\Delta} & =\langle x(t), x(t)\rangle^{\Delta}=\left\langle x(t)+x(\sigma(t)), x^{\Delta}(t)\right\rangle \\
& =\left\langle x(t)+x(t)+\mu(t) x^{\Delta}(t), x^{\Delta}(t)\right\rangle=2\left\langle x(t), x^{\Delta}(t)\right\rangle+\mu(t)\left\|x^{\Delta}(t)\right\|^{2} .
\end{aligned}
$$

The above conclusion leads us to another definition of OSP on a time scale. Namely, $F(t, \cdot)$ is said to be OSP if there exists a Perron function $V(\cdot, \cdot)$ such that, for every $f_{x} \in$ $F(t, x)$, there exists $f_{y} \in F(t, y)$ such that

$$
2\left\langle x-y, f_{x}-f_{y}\right\rangle+\mu(t)\left|f_{x}-f_{y}\right|^{2} \leq V\left(t,|x-y|^{2}\right) .
$$

In this case, however, it is difficult to prove a meaningful version of the Filippov-Pliss lemma, although the following theorem is true.

Theorem 4.6 Let $x(\cdot)$ be a solution of (3.1) with $x\left(t_{0}\right)=x_{0}$. Then, for any $y_{0}$, there exists a solution $y(\cdot)$ of (3.1) with $y\left(t_{0}\right)=y_{0}$ such that

$$
|x(t)-y(t)|^{2} \leq r(t), \text { where } r\left(t_{0}\right)=\left|x_{0}-y_{0}\right|^{2} \quad \text { and } \quad r^{\Delta}(t)=V(t, r(t)) .
$$

Example 4.7 Let $\mathbb{T}$ be a time scale on $[0,1]$. Let $\left\{x_{i}\right\}_{i}$ be a dense subset of $\mathbb{B}$. Define the multifunction $H(x)=\overline{c o}\left\{f_{i}(t, x)\right\}_{i=1}^{k}+\sum_{i=k}^{\infty} \frac{f_{i}(t, x)}{2^{i}}$, where $k \geq 5, f_{i}(t, x)=c(t) g_{i}(x)$ and

$$
g_{i}(x)= \begin{cases}-\frac{x-x_{i}}{\sqrt{\left|x-x_{i}\right|}}, & x \neq x_{i}, \\ 0, & x=x_{i} .\end{cases}
$$

While $c=\max \{\tau-t:[t, \tau] \subset \mathbb{T}\}$. Clearly every $g_{i}(\cdot)$ is one-sided Lipschitz with a constant 0 . Therefore $H(t, \cdot)$ is OSL with a constant 0 on any point $(t, x)$ with $t \in \mathbb{T}_{\text {rd }}$ and it is 0 on any point $(t, x)$ with $t \in \mathbb{T}_{\mathrm{rs}}$.

Let $G: \mathbb{T} \times \mathbb{R}^{n} \rightrightarrows \mathbb{R}^{n}$ be bounded full Perron. Define $F(t, x)=H(x)+G(t, x)$. Then $F(\cdot, \cdot)$ satisfies all the assumptions of Theorem 4.2.

Then clearly system (3.1) with $t_{0}=0, T=1$ and $x_{0}=0$ satisfies the conditions of Theorem 4.4.

Now we discuss the closure of the solution set for system $(3.1)$ when $F(\cdot, \cdot)$ is almost continuous and not necessarily convex-valued. Notice that we only show some further studying directions.

Clearly the closure of the solution set of (3.1) is not the solution set of

$$
x^{\Delta}(t) \in \overline{c o} F(t, x), \quad x\left(t_{0}\right)=x_{0} .
$$

The following theorem holds true. 
Theorem 4.8 Let $F(\cdot, \cdot)$ be almost continuous with compact values. Suppose that $\mathbf{F} 1$ holds. The closure of the solution set of (3.1) is a subset of the solution set of

$$
x^{\Delta}(t) \in H(t, x(t)), \quad x\left(t_{0}\right)=x_{0},
$$

where

$$
H(t, x)= \begin{cases}\overline{c o} F(t, x), & t \in \mathbb{I}_{\mathrm{rd}}, \\ F(t, x), & t \in \mathbb{I}_{\mathrm{rs}} .\end{cases}
$$

Proof Let $x_{m}(\cdot)$ be a sequence of solutions of (3.1) such that $x_{m}(t) \rightarrow x(t)$ uniformly on $\mathbb{I}$. As in [9] we can extend every $x_{k}^{\Delta}(\cdot)$ on $I$ as a Lebesgue integrable function $g_{k}(\cdot)$. Due to Diestel criterion (see, e.g., [27]), the sequence $\left\{g_{k}(\cdot)\right\}_{k}$ is weakly $L_{1}$ precompact and passing to subsequences if necessary $g_{k}(t) \rightarrow g(t) L_{1}$ weakly. Due to Mazur's lemma, there exists a convex combination $\sum_{i=k}^{k_{i}} \alpha_{i} g_{i}(t)$ converging to $g(t) L_{1}$ strongly and passing to subsequences for a.a. $t \in I$. Clearly its restriction to $\mathbb{I} g_{\mathbb{I}}(t) \in \overline{c o} F(t, x(t))$ for $\Delta$ a.e. $t$. Notice that every $\left.g_{(}\right)$is constant on $[t, \sigma(t)]$, the latter is not a single point in the case $t \in \mathbb{I}_{\mathrm{rs}}$. Since $\mathbb{I}_{\mathrm{rs}}$ is countable, then it is easy to show that in $g(t) \in F(t, x(t))$ for $\Delta$ a.a. $t \in \mathbb{I}_{\mathrm{rs}}$. Consequently, $g(t) \in H(t, x(t))$.

It will be interesting to prove or disprove the following conjecture, which is an analogue of the very important in the optimal control relaxation theorem.

Conjecture 1 Let $F(\cdot, \cdot)$ be almost continuous with compact values. Suppose that $\mathbf{F 1}$ holds and $F(t, \cdot)$ is OSP. Then the closure of the solution set of (3.1) is the solution set of (4.2).

We hope that the reader will be able to prove this conjecture.

\section{Conclusion}

In this paper we prove a Filippov-Pliss type theorem for time scale dynamical inclusion, when the right-hand side satisfies the well-known Lipschitz condition on the state variable. Afterward we extend the one-sided Perron condition to time scale systems. The FilippovPliss lemma is then extended to OSP dynamical inclusions.

Notice that we have to distinguish the right dense and right scattered points in the Filppov-Pliss lemma and relaxation theorem. The conditions are different than in the case of continuous systems.

We prove also the existence of solutions to so-called almost LSC dynamical inclusions and pose a conjecture that a variant of the so-called relaxation theorem is true.

Now we discuss some open problems.

\subsection{Almost LSC problem}

It seems that the following result is true.

Proposition 5.1 Let $F(\cdot, \cdot)$ be almost LSC with closed nonempty values. Under F1 the dynamical inclusion (3.1) admits a solution. 
Proof (Idea). Consider the compacts $\mathbb{I}_{k}$ as in the previous proof, where $F(\cdot, \cdot)$ is LSC on $\mathbb{I}_{k} \times \mathbb{R}^{n}$. Let $N$ be from Remark 3.2. Consider the cone $K_{N}=\left\{(t, x) \in \mathbb{I} \times \mathbb{R}^{k}:|x| \leq(N+1) t\right\}$.

It is well known that every LSC multifunction has a $\Gamma^{N+1}$ continuous selection, i.e., selection $f(t, x) \in F(t, x)$ such that $f_{k}\left(t_{i}, x_{i}\right) \rightarrow f_{k}(t, x)$ if $\mathbb{I}_{k} \ni t_{i} \rightarrow t$ and $\left|x_{i}-x\right| \leq(N+1)\left(t_{i}-t\right)$ (see, e.g., Lemma 6.2 in [28]).

Define the multifunction $G_{k}(t, x)=\bigcap_{\varepsilon>0} \overline{c o} f_{k}\left((t-\varepsilon, t+\varepsilon) \cap \mathbb{I}_{k}, x+\varepsilon \mathbb{B}\right)$. Clearly $G_{k}(\cdot, \cdot)$ is USC on $\mathbb{I}_{k} \times \mathbb{R}^{n}$. Let $G(t, x)=G_{k}(t, x)$ as $t \in \mathbb{I}_{k}, k=1,2, \ldots$, and $G(t, x)=\{0\}$ otherwise (see, e.g., [29]). Consequently, $G(\cdot, \cdot)$ is almost USC, and hence the dynamical inclusion

$$
x^{\Delta}(t) \in G(t, x(t)), \quad \Delta \text {-a.e. } t \in \mathbb{I}, x\left(t_{0}\right)=x_{0}
$$

has a solution $y(\cdot)$.

Dealing as in the proof of Lemma 6.1 of [28] (see also [29]), one can try to prove that $y(\cdot)$ is also a solution (3.1). The obstacle is that we work with right dense points.

\section{Acknowledgements}

The authors would like to thank all the anonymous reviewers and the auditors for their helpful advice and hardwork. The work was supported by the University of Lahore, Lahore, Pakistan.

\section{Competing interests}

The authors declare that they have no competing interests.

\section{Authors' contributions}

RA, TD and VL contributed to Sections 1, 2, 3 and MR contributed to Section 4. All authors read and approved the final manuscript

\section{Author details}

'Department of Mathematics, University of Lahore, Lahore, Pakistan. ${ }^{2}$ Abdus Salam School of Mathematical Sciences, 68-B, New Muslim Town, Lahore, Pakistan. ${ }^{3}$ Department of Mathematics, "Al. I. Cuza" University, laşi, 700506, Romania. ${ }^{4}$ University Constantin Brâncuşi, Str. Geneva, Nr. 3, Târgu-Jiu, 210136, Romania.

\section{Publisher's Note}

Springer Nature remains neutral with regard to jurisdictional claims in published maps and institutional affiliations.

Received: 20 May 2017 Accepted: 12 September 2017 Published online: 29 September 2017

\section{References}

1. Hilger, S: Ein Maßkettenkalkül mit Anwendungauf Zentrumsmanningfaltigkeiten. Doktorthesis, UniversitätWürzburg (1988)

2. Hilger, S: Analysis on measure chains - a unified approach to continuous and discrete calculus. Results Math. 18, 18-56 (1990)

3. Bohner, M, Peterson, A: Dynamic Equations on Time Scales: An Introduction with Applications. Birkhäuser, Boston (2001)

4. Bohner, M, Peterson, A: Advances in Dynamic Equations on Time Scales. Birkhäuser, Boston (2003)

5. Agarwal, R, Bohner, M, O'Regan, D, Peterson, A: Dynamical inclusions on time scale: a survey. J. Comput. Appl. Math. $141,1-26(2002)$

6. Bohner, M, Davis, J: Dynamical equations on time scale: qualitative analysis and applications. Nonlinear Dyn. Syst. 9 (2009)

7. Laksmikantham, V, Sivasundram, S, Kaymakcalan, B: Dynamic Systems on Measure Chains. Kluwer Academic, Dordrecht (1996)

8. Guseinov, G: Integration on time scales. J. Math. Anal. Appl. 285, 107-127 (2003)

9. Cabada, A, Vivero, D: Expression of Lebesgue $\Delta$ integral on time scales as a usual Lebesgue integral: application to the calculus of $\Delta$-antiderivatives. Math. Comput. Model. 43, 194-207 (2006)

10. Liu, G, Xiang, X, Peng, Y: Nonlinear integro-differential equations on time scales. Comput. Math. Appl. 61, 159-169 (2011)

11. Miranda, F: Differential inclusions and optimization problems on time scales. In: Num. Anal. Appl. Math. ICNAAM 2012 AlP Conf. Proc, vol. 1479 (2012). doi:10.1063/1.4756243

12. Peng, Y: A class of optimal control problems on time scale. Energy Proc. 16, 1760-1767 (2012)

13. Peng, Y, Xiang, $X$, Jaangy, Y: Nonlinear dynamical systems and optimal control on time scale. ESAIM Control Optim. Calc. Var. 17, 654-681 (2011)

14. Atici, F, Biles, D: First order dynamical inclusions on time scale. J. Math. Anal. Appl. 292, 222-237 (2004) 
15. Frigon, M, Gilbert, H: Systems of first order inclusions on time scale. Topol. Methods Nonlinear Anal. 37, 147-163 (2011)

16. Santos, I, Silva, G: Absolute continuity and existence of solutions of dynamical inclusions in time scale. Math. Ann. 356, 373-399 (2013)

17. Santos, I, Silva, G: Filippov's selection theorem and the existence of solutions for optimal control problems on time scale. Comput. Appl. Math. 33, 223-241 (2014)

18. Donchev, T, Farkhi, E: On the theorem of Filippov-Pliś and some applications. Control Cybern. 38, 1251-1271 (2009)

19. Filippov, A: Classical solutions of differential equations with multivalued right-hand side. SIAM J. Control Optim. 5 , 609-621 (1967)

20. Pliss, A: On trajectories of orientor fields. Bull. Acad. Pol. Sci., Sér. Sci. Math. Astron. Phys. 135, 571-573 (1965)

21. Aubin, J-P, Frankowska, H: Set-Valued Analysis. Birkhäuser, Boston (1990)

22. Lempio, F, Veliov, V: Discrete approximations of differential inclusions. Bayreuth. Math. Schr. 54, 141-232 (1998)

23. Blagodatski, V, Filippov, A: Differential inclusions and optimal control. Proc. Steklov Inst. Math. 4, $199-259$ (1986)

24. Donchev, T, Farkhi, E: Stability and Euler approximation of one-sided Lipschitz differential inclusions. SIAM J. Control Optim. 36, 780-796 (1998)

25. Hu, S, Papageorgiou, N: Handbook of Multivalued Analysis Volume I Theory. Kluwer Academic, Dordrecht (1997)

26. Smirnov, G: Introduction to the Theory of Differential Inclusions. Am. Math. Soc., Providence (2002)

27. Diestel, J, Ruess, W, Schachermayer, W: Weak compactness in $L^{1}(\mu, X)$. Proc. Am. Math. Soc. 118, 447-453 (1993)

28. Deimling, K: Multivalued Differential Equations. de Gruyter, Berlin (1992)

29. Cernea, A, Stuicu, V: Directionally continuous selections and nonconvex evolution inclusion. Set-Valued Anal. 11, 9-20 (2003)

\section{Submit your manuscript to a SpringerOpen ${ }^{\circ}$ journal and benefit from:}

- Convenient online submission

- Rigorous peer review

- Open access: articles freely available online

- High visibility within the field

- Retaining the copyright to your article

Submit your next manuscript at $\gg$ springeropen.com 